\title{
Alterstice
}

Revue internationale de la recherche interculturelle

International Journal of Intercultural Research

Revista International de la Investigacion Intercultural

\section{Congrès à Halifax et lancement du prix ARIC 2019}

\section{Bureau de l'Association internationale pour la recherche interculturelle (ARIC)}

Volume 8, numéro 1, 2018

URI : https://id.erudit.org/iderudit/1052602ar

DOI : https://doi.org/10.7202/1052602ar

Aller au sommaire du numéro

Éditeur(s)

Alterstice

ISSN

1923-919X (numérique)

Découvrir la revue

Citer ce document

Bureau de l'Association internationale pour la recherche interculturelle (ARIC) (2018). Congrès à Halifax et lancement du prix ARIC 2019. Alterstice, 8(1), 3-4.

https://doi.org/10.7202/1052602ar

Droits d'auteur (C) Bureau de l'Association internationale pour la recherche interculturelle (ARIC), 2018
Ce document est protégé par la loi sur le droit d'auteur. L'utilisation des services d'Érudit (y compris la reproduction) est assujettie à sa politique d'utilisation que vous pouvez consulter en ligne.

https://apropos.erudit.org/fr/usagers/politique-dutilisation/ 


\section{ᄀ \\ alterstice}

RUBRIQU' ARIC

\section{Congrès à Halifax et lancement du prix ARIC 2019}

Bureau de l'Association internationale pour la recherche interculturelle (ARIC) ${ }^{1}$

L'ARIC est dynamique dans divers réseaux et participe au développement de la recherche interculturelle du Sud au Nord. Au travers de partenariats avec des groupes de recherche ou avec des universités, l'association soutient des colloques, conférences ou séminaires dans divers pays et autour de thématiques pertinentes pour le domaine interculturel.

C'est ainsi que nous avons été partenaires du colloque international Immigration, dynamiques identitaires et politiques de gestion de la diversité, qui s'est tenu à Ste Mary's University à Halifax en Nouvelle-Écosse (Canada) en juin 2018. Nous avons été plusieurs membres de l'ARIC à y participer et nous avons apprécié la qualité scientifique, la pertinence, les échanges et l'organisation de ce colloque bilingue français-anglais. En tant qu'association francophone, nous tenons d'abord à féliciter l'équipe organisatrice pour ses efforts de traduction des conférences dans les deux langues. Les présentations figurent d'ailleurs maintenant sur le site Internet du colloque en français et en anglais.

En ce qui concerne l'organisation du colloque, il est important de noter la multidisciplinarité des interventions et des participants, parmi lesquels des représentants des milieux associatifs, institutionnels et politiques, ainsi que l'intérêt de tenir des panels, tables rondes et aussi des ateliers permettant d'approfondir et d'expérimenter certaines thématiques comme les médiations interculturelles, l'évaluation du climat interculturel en milieu scolaire ou la question des sources de données concernant l'immigration.

Le contenu des présentations a permis de balayer tant le champ conceptuel que le domaine des pratiques et politiques en ce qui concerne l'immigration, les questions identitaires qui y sont souvent associées et les modèles de gestion de la diversité. Plusieurs conférences et ateliers portaient sur les réalités politiques et migratoires du Canada, avec des présentations de participants venant de France, du Brésil, de Finlande, des États-Unis, d'Égypte ou d'organismes internationaux, ce qui a permis d'introduire une perspective internationale fort importante pour la réflexion et les avancées de la recherche interculturelle.

Les analyses et concepts très contemporains de tension entre cosmopolitisme et nationalismes, entre société ouverte et société fermée ainsi que les phénomènes de doutes et de crispations identitaire ont été abordés au travers de réalités parfois canadiennes, comme la fusillade à la mosquée de Québec, parfois transnationales comme la question des étudiants internationaux dans divers pays, la solidarité internationale dans les ONG humanitaires de santé, l'émergence des universités interculturelles dans les milieux autochtones ou encore le travail social international. Les politiques d'immigration, d'intégration et de gestion de la diversité ont fait l'objet d'analyses critiques et de questionnements tant conceptuels que sur le plan de leurs résultats et effets pervers. On a ainsi abordé les questions de la sélection des immigrants, de la place des réfugiés, du racisme et des discriminations ou encore du genre et de la violence dans les migrations forcées. Finalement, les processus de communication, de compétences et de relations interculturelles ont été abordés, analysés et comparés dans divers milieux et pays : dans l'enseignement, dans la gestion et l'emploi, dans les réseaux virtuels ou encore dans les organisations pour la petite enfance, etc. Sur le plan méthodologique, notons l'émergence de tout un courant de pensée et d'analyse qui met la narration, l'art, le dialogue et l'écriture biographique au cœur de la recherche et des nouvelles pratiques et politiques.

Le colloque, avec son accueil chaleureux et son climat interculturel et convivial, a sans aucun doute permis à toutes les participantes et tous les participants, universitaires comme professionnels de terrain, d'avancer sur 
le thème de l'interculturalité, de partager travaux de recherche et savoir-faire tout en développant des projets de travail collaboratif entre chercheurs et acteurs sociaux.

Et les collaborations ne s'arrêteront pas là. L'équipe organisatrice du colloque d'Halifax est invitée à proposer des publications dans la collection de l'ARIC, à devenir membre, à participer au prochain congrès à Genève. De même, I'ARIC continuera son partenariat dans l'organisation et la diffusion des prochaines activités et colloques du réseau mis en œuvre par l'Université Ste Mary de Nouvelle Écosse et l'Université Laval de Québec.

Si l'ARIC se développe ainsi de manière horizontale et internationale par ses partenariats divers, elle ne s'arrête pas là. Le soutien à la relève, dans une optique de transmission des connaissances et d'émergence de nouveaux savoirs, se concrétise par le prix de la meilleure thèse de doctorat en recherche interculturelle remis lors de chaque congrès de I'ARIC depuis 2013. La quatrième édition de ce prix vient d'être lancée. Le concours s'adresse à toute personne ayant terminé et soutenu avec succès une thèse de doctorat en recherche interculturelle dans les deux ans qui précèdent le congrès qui aura lieu en juin 2019 (soit un dépôt et une soutenance entre janvier 2017 et novembre 2018).

La thèse peut relever de différentes disciplines des sciences humaines et sociales : anthropologie, didactiques de langues étrangères, géographie, histoire, linguistique, philosophie, psychologie, sciences de l'éducation, sciences politiques, sciences économiques, sociologie, travail social. Une nouveauté pour 2019 : l'appel à candidature pour ce prix s'adresse en priorité aux thèses rédigées en français, langue officielle de l'ARIC. Peuvent également entrer en ligne de compte des thèses rédigées dans d'autres langues (à l'exception toutefois de l'anglais), pour autant que l'ARIC dispose d'évaluateurs ou évaluatrices dans la langue concernée. C'est pourquoi les candidats qui souhaitent soumettre une thèse rédigée dans une autre langue doivent commencer par prendre contact avec le secrétariat de I'ARIC, afin de vérifier si une candidature est possible dans la langue envisagée. Dans tous les cas, le résumé associé à la candidature et la lettre de candidature doivent être rédigés en français. En outre, le lauréat ou la lauréate doit être en mesure d'assurer une communication en français lors du congrès 2019.

La candidature doit être présentée avec le dossier complet au plus tard le 30 novembre 2018, à l'adresse électronique du secrétariat de I'ARIC : info@aric-interculturel.com.

Les dossiers seront évalués par le comité des sages de l'ARIC et des évaluateurs désignés afin que la recommandation pour la candidature retenue soit effective en mars 2019 et permette une participation au congrès 2019 à Genève. L'ARIC attend des candidatures nombreuses et diversifiées à ce concours et sera fière de remettre en 2019 ce prix à un nouveau chercheur ou à une nouvelle chercheure participant au développement, à la reconnaissance et à la l'approfondissement de la recherche interculturelle.

Pour toute information sur le colloque international d'Halifax, consulter le site International Conference in Intercultural Studies à https://www.icstconference.com

Pour toutes informations sur le prix ARIC de la meilleure thèse en recherche interculturelle, consulter le site de I'ARIC à http://www.aric-interculturel.com/prix-aric/

\section{Rattachement des auteurs}

1 Les auteurs constituent le bureau de l'Association internationale pour la recherche interculturelle.

\section{Correspondance}

info@aric-interculturel.com

\section{Pour citer cet article}

Bureau de l'ARIC. (2018). Congrès à Halifax et lancement du prix ARIC 2019 [Rubriqu'ARIC]. Alterstice, 8(1), 3-4. 